\title{
Peculiarities of control of erythrocytes moving in an evanescent field
}

\author{
Oleg V. Angelsky \\ Peter P. Maksymyak \\ Claudia Y. Zenkova \\ Andrew P. Maksymyak \\ Steen G. Hanson \\ Dimitrov D. Ivanskyi
}




\title{
Peculiarities of control of erythrocytes moving in an evanescent field
}

\author{
Oleg V. Angelsky, ${ }^{\mathrm{a}, \mathrm{b}, \star}$ Peter P. Maksymyak, ${ }^{\mathrm{a}}$ Claudia Y. Zenkova, ${ }^{\mathrm{a}}$ Andrew P. Maksymyak, ${ }^{\mathrm{a}}$ Steen G. Hanson, ${ }^{\mathrm{c}}$ \\ and Dimitrov D. Ivanskyi ${ }^{\mathrm{a}}$ \\ ${ }^{a}$ Chernivtsy National University, Chernivtsy, Ukraine \\ 'Taizhou Research Institute of Zhejiang University, Taizhou, China \\ 'DTU Fotonik, Department of Photonics Engineering, Roskilde, Denmark
}

\begin{abstract}
An investigation of the influence of an evanescent wave on the dynamics of motion of erythrocytes in blood plasma is presented. Computer simulation of erythrocytes moving in an evanescent field and experimental demonstration of the forecasted motion substantiate the possibility for control of position of red blood cells in a solution. The range of velocities of transversal motion of erythrocytes due to the action of the optical force of the generated evanescent field is determined as a function of the angle of illumination of a cell by a linearly polarized wave with the azimuth of polarization $45 \mathrm{deg}$. $\odot$ The Authors. Published by SPIE under a Creative Commons Attribution 4.0 Unported License. Distribution or reproduction of this work in whole or in part requires full attribution of the original publication, including its DOI. [DOI: 10.1117/1 .JBO.24.5.055002]
\end{abstract}

Keywords: erythrocytes; evanescent wave; spin momentum.

Paper 180491RR received Aug. 16, 2018; accepted for publication Apr. 17, 2019; published online May 20, 2019.

\section{Introduction}

A majority of investigations in biology and medicine carried out over the last decades are devoted to development of methods for studying biological objects with visualization of micromanipulations at molecular and cellular scales. Noninvasive optical trapping and moving the microobjects presume application of approaches characterized by superhigh accuracy ${ }^{1}$ used for investigations of biomarkers, ${ }^{2}$ DNA molecules,${ }^{3,4}$ myosin,,${ }^{5}$ kinesin, ${ }^{6}$ etc. As a rule, controlled manipulations of microobjects are performed using optical tweezers. (This approach and term have been proposed by Ashkin in 1970.7) At present, a set of techniques is available for manipulation of biological objects and microparticles using optical and magnetic tweezers, as well as atom-force microscopy and raysounding of cells. Specifically, the optical techniques provide noninvasive, contactless trapping of microparticles or organelles in cells, by transfer of the linear momentum from a photon. Optical tweezers are used as the primary tool in biophysical investigations ${ }^{8}$ for measuring the dissolving, studying kinetochore-microtubule interaction ${ }^{9}$ and quantifying intercellular interactions. ${ }^{10}$ Traditionally, an optical trap is a sharply focused laser beam formed by a microscope objective with a high numerical aperture, which enables control of position and motion of microobjects by applying optical force. The magnitude of the optical force is on the order of a few $\mathrm{pN}$, and the physical mechanism of the action of this force creates the interaction of EM radiation with the induced dipole momentum of the dielectric particles. Transfer of the linear momentum from light to particles depends on the optical properties of the controlled microparticles and on the phase distribution and polarization of the probing beam.

Taking into account optical diffraction, the study of smallsized systems is delicate due to limitation on the maximal

*Address all correspondence to Oleg V. Angelsky, E-mail: o.angelsky@chnu .edu.ua magnitude of the energy density achieved in optical tweezers for a fixed radiation power. This limitation is of importance for biological samples, which can be destroyed by high-power radiation

Widespread plasmonic optical tweezers, ${ }^{11}$ so-called plasmonic nanotubes with metallic nanoantennas, are used for amplification and concentration of energy of optical fields within deep subwavelength gaps. This provides high magnitudes of the intensity gradient in the near field and results in growing optical forces for a fixed input radiation power, being lower than the threshold for biomaterial damage by two to three orders of magnitude.

Therefore, it is of importance to search for techniques for manipulation of biological microobjects at the cell scale in vivo, by the use of physiological permeability of biomembranes. The use of optical tweezers for trapping and manipulating erythrocytes in blood capillaries makes it possible to clean microvessels and renew their permeability owing to combination of the results of biological investigations in vitro and in vivo. ${ }^{12}$ The ability of laser radiation to penetrate biological tissues enables creation of systems for noninvasive manipulation of biological micro- and nano-objects in living organisms, including realization of controlled spatial motion of such objects, while avoiding tissue destruction.

Here, we investigate the influence of laser radiation on erythrocytes (red blood cells, RBCs), both for optical trapping and moving, and for realization of precise control of such objects at the nanoscale level, providing desirable velocity of motion in the evanescent field due to changing polarization of a laser beam and change in illumination conditions. This approach enables control of blood vessels using low-power laser systems and gradual renewal of blood supplying necrotic tissues. Evanescent waves excited above the interface of two media with different refraction indices are the complementary source of optical forces that can be employed for manipulation of microparticles in a broader range of applications than conventional tweezers. 
Note, special attention is paid to waveguide structures, optical fibers, and optical fiber-based structures such as trapper, subwavelength wire, and fiber-ring, providing control and capturing of isolated molecules, microspheres, ${ }^{13}$ and massive dielectric particles. ${ }^{14,15}$ Investigations in $2011^{15}$ have demonstrated the possibility of using a defect-decorated optical nanofiber for targeted delivery of nanoparticles. Progress of diagnostic and therapeutic techniques used in practical medicine promotes further search for tools for controlled movement of nano-objects, including RBCs in biological systems.

Developing the approaches proposed in the given paper, one can either look for the conditions for the realization of an evanescent wave at the interface of the derma-blood vessel (RBS), thereby providing noninvasive methods for controlling the erythrocyte motion, or taking invasive intervention with a pointed rectangular fiber and correctly selected refraction index. This facilitates the realization of vertical spin of the evanescent wave for erythrocyte manipulation.

Thus, the purpose of this work is to study the influence of the optical evanescent wave on erythrocytes, including experimental demonstration of controlled location and motion of a cell, depending on the illumination conditions.

\section{Simulation of Interaction of Erythrocytes with an Evanescent Wave}

Intense investigations of the optical forces acting on micro- and nanoparticles localized near interface of two media are carried out here for elucidating the distribution of the components of an optical field above a microstructured surface. Complex geometry of a surface and surface inhomogeneities may considerably reinforce the optical forces and cause appearance of new components connected, in part, with manifestation of the extraordinary vertical spin angular momentum of an evanescent wave. ${ }^{16}$ Miniaturization of the optical traps leads to creation of controlled microspheres, which act as micropumps and switches. From this standpoint, the paper ${ }^{17}$ is of special interest devoted to the study of the behavior of microparticles located at the distances of a few micrometers from a surface, viz. at the evanescent field where one must take into account both optical and hydrodynamic surface effects. Taken together, this provides the description of complex motion of the particles captured by an evanescent wave in two-dimensional representation, with formation the trace as an elliptic form at the plane of extinction of an evanescent wave.

Meanwhile, in the case of isolated erythrocytes, the question on feasibilities for controlled motion of these cells using an evanescent wave excited at the boundary "prism-blood plasma" remains unsolved. To enhance the feasibilities for noninvasive control of RBCs using laser radiation, we propose to excite an evanescent wave using a linearly polarized wave with an azimuth of polarization $45 \mathrm{deg}$, enabling realization of transversal spin flows in the evanescent wave and controlled spatial (transversal) motion of erythrocytes.

As a rule, various biological microobjects can be localized in different media. The control of such microobjects calls for the use of a large set of optical forces to provide directional action on the controlled object. These forces can be evaluated within the framework of the relevant optical theory. Theoretical models and experimental approaches approved up to now enable prognosing the behavior of objects of different shape, such as spheres, cylinders, and objects of more complex shapes, in optical traps and tweezers.
Here, we focus our attention on erythrocytes. The normal human erythrocyte has the shape of concavo-concave disc. The diameter of an erythrocyte is $7.82 \pm 0.62 \mu \mathrm{m}$, and its minimal and maximal thickness at the center and at the limb are $0.81 \pm 0.35$ and $2.58 \pm 0.27 \mu \mathrm{m}$, respectively. The mean magnitude of the cell volume of an erythrocyte is $94 \pm 14 \mu \mathrm{m}^{3}$, and the mean magnitude of the cell surface area is $135 \pm 16 \mu \mathrm{m}^{2}$. The mentioned sizes of an erythrocyte are estimated from measurings on photomicrographs. ${ }^{18}$ The model of concavo-concave disc corresponds well to native erythrocytes experimentally proven. ${ }^{19}$ Erythrocyte can be deformed in a shearing flow, where its shape becomes ellipsoidal. ${ }^{20}$ Considering an erythrocyte as a uniforn disc with the mean erythrocyte volume, one can use the concept of single light-scattering by a cell where the cell organelles and the cell membrane are believed to be thin enough to neglect their influence of its real thckness on the light scattering properties. The Mie theory of light scattering applied for such cases provides a comprehensive description of the energy transfer to the erythrocyte. ${ }^{18}$

- Modeling of an erythrocyte by a disc facilitates the determination of an algorithm that represents a cell in an evanescent. We are interested in understanding the transversal motion of such a cell. This becomes possible, if the probing wave is linearly polarized, with the azimuth of polarization $\pm 45 \mathrm{deg}$. An elliptically polarized evanescent incident wave introduces a vertical spin angular momentum. This introduces transversal and longitudinal forces, inducing motion of the erythrocyte.

In contrast to previous studies of induced longitudinal motion induced by an evanescent field, we will here study transversal motion. This motion may be introduced by a combination of the incident field and its polarization. Therefore, rotational motion of an erythrocyte is not the subject of study in this work, while such motion has been investigated and experimentally proven. ${ }^{21}$

Let us consider the theoretical model for exciting the transversal spin of an evanescent wave, as well as the densities of the spin and orbital flows compared with the experimentally observed rectilinear motion of a cell.

Observation of motion of isolated erythrocytes in whole blood is hampered by high concentration of cells. Concentration of erythrocytes is diminished using an isotonic solution. However, the simplest use of physiological solution is not practicable due to the lower viscosity of the solution changing the elasticity of erythrocytes. Consequently, we diminished the erythrocyte concentration using the natural medium of cells, viz. a blood plasma. Following this approach, both computer simulation and experimental demonstration of erythrocyte motion are carried out in blood plasma with refractive index $\left(n_{\text {sol }}=1.37\right)$. To realize the total internal reflection, we use a flint prism with an index of refraction $n_{\mathrm{pr}}=1.61$, above which an erythrocyte with the refraction index $n_{\mathrm{er}}=1.407$ is located ${ }^{18}$ in the blood plasma. To avoid the temperature effects and irreversible temperature changes due to heating and destroying cells under illumination, one sets the power of radiation below $100 \mathrm{~mW}$ and the pulse cycling of about $1 \mu \mathrm{s}$.

An evanescent wave (Fig. 1, III) formed above the prism surface (Fig. 1, 1) penetrates into the erythrocyte (Fig. 1,3) and creates an inhomogeneous intensity distribution in the $X Z$ plane, which is connected with the penetration depth for the 


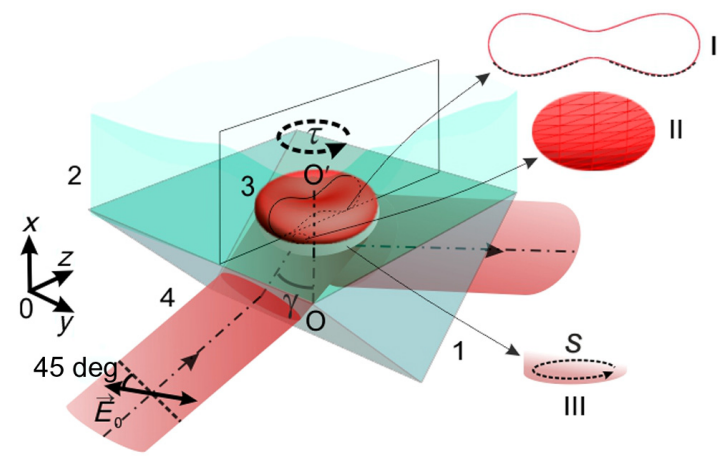

Fig. 1 Arrangement for realization of rotational motion of an erythrocyte (3) in the evanescent wave (III). S: spin angular momentum of an evanescent wave; $\tau$ determines the direction of the torque on the erythrocyte (3) localized in blood plasma (2) above the prism surface (1). $\gamma$ : the angle of incidence of linearly polarized light wave (4) $\vec{E}_{0}$ with the azimuth of polarization $45 \mathrm{deg}$ with respect to the plane of incidence at the interface "prism-liquid," where the conditions of the total internal reflection are fulfiled. Inset (I): transversal cross-section of an erythtocyte with depicted facets used for simulation ( $N$ being the total number of facets). Lower inset (II): image of the area of an erythrocyte surface (1:64) with depicted facets in the XOZ-plane.

corresponding evanescent wave $d_{x}=\left[\frac{\lambda}{2 \pi n_{\mathrm{pr}} \sqrt{\sin ^{2} \gamma-\left(\frac{n_{\mathrm{sol}}}{n_{\mathrm{pr}}}\right)^{2}}}\right]$. The the angle of incidence of the beam at the prism surface is $\gamma$. By changing the angle of incidence and the wavelength, one can control this depth up to $0.9 \mu \mathrm{m}$. For the azimuth of polarization of the incident wave $\pm 45 \mathrm{deg}$, the evanescent wave, being elliptically polarized, has a torque $(\tau)$ causing rotation of an erytrhocyte (Fig. 1).

The geometric parameters of an erythrocyte, ${ }^{22,23}$ used for modeling the cell shape are: $D=7.2 \mu \mathrm{m} ; h=2.1 \mu \mathrm{m}$; $b=0.9 \mu \mathrm{m} ; c=4.74 \mu \mathrm{m}$ (Fig. 2).

Estimations for objects of complex shape (such as an erythrocyte) through simple analytic computations of interaction of an evanescent wave and a cell are difficult, ${ }^{23}$ being connected with considerable errors. Therefore, the following approach is proposed for modeling of an erythrocyte and determination of the conditions of interaction of a cell with an evanescent wave, here describing the erythrocyte shape as the set of facets of equal areas $0.01 \mu \mathrm{m}^{2}$. The chosen size of the facets is necessary and sufficient for the description of the erythrocyte surface interacting with an evanecent wave. In respect to the transversal size $h$ of an erythrocyte, $1 / 3 h$ takes part in the interaction of the cell and the evanescent wave. One computes the components of the optical force $F_{x}, F_{y}, F_{z}$ and the magnitude of torque $\tau$ for each facet. In doing so, one takes into account the angle of incidence of radiation at the corresponding facet and optical characteristics of the erythrocyte surface. Computer simulations were carried out for 3340 facets interacting with an evanescent

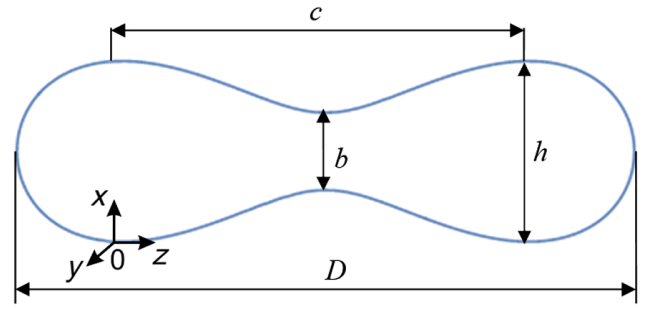

Fig. 2 Transversal cross-section of an erythrocyte. $D, h, b, c$ : parameters of an erythrocyte used in simulation. wave adding up the derived components of the optical force for three directions for each facet. In the same way, one computes the resulting magnitude of torque. During simulation, the coordinates are obtained from the following equation describing the shape of an erythrocyte as a concavo-concave disc: ${ }^{22}$

$$
\left(y^{2}+z^{2}\right)^{2}+2 S\left(y^{2}+z^{2}\right) x^{2}+x^{4}+P\left(y^{2}+z^{2}\right)+Q x^{2}+R=0 .
$$

Correspondingly, the parameters $P, Q, R$, and $S$ can be determined as

$$
\begin{aligned}
& P=-\frac{D^{2}}{4}-\frac{b^{2} h^{2}}{4 D^{2}}+\frac{b^{2} c^{4}}{4 D^{2}\left(h^{2}-b^{2}\right)}, \\
& Q=\frac{D^{4}+4 D^{2} P-b^{4}}{4 b^{2}}, \\
& R=-\frac{D^{4}}{16}\left(D^{2}+4 P\right), \\
& S=-\frac{c^{2}+2 P}{h^{2}} .
\end{aligned}
$$

Let us set the coordinates $X Y Z$ with the origin at point $O$ $(0,0,0)$, as it is shown in Fig. 2. The coordinates of facets are measured from this point to right and to left along the axes $O Y$ and $O Z$ with the stepsize $0.01 \mu \mathrm{m}$. This step size provides the optimized condition for computer simulation. Following Eq. (1), for each $(y, z)$ position, one determines the magnitude $x$ for the point at the erythrocyte surface. The areas of the facets chosen for simulation $\left(\sim 0.01 \mu \mathrm{m}^{2}\right)$ are determined using computer fitting. The upper magnitude of the coordinate along the $X$ axis is chosen in correspondence with the preliminary estimated depth of penetration of an evanescent wave into the erythrocyte. The use of software Mathematica enables such division in triangular facets of equal areas, with determination of the apex of each facet, which is used for simulation of the erythrocyte surface. Using the mentioned software, one can estimate the coordinates of the each point of a facet, as well.

The obtained set of facets is distinct in its spatial position in the specified coordinates $X Y Z$ (Fig. 3). Each facet is marked by its normal orientation and by the angle of inclination of a facet, $\alpha_{i}$, to the $Y O Z$ plane. In addition, the facets are also different in the angle of rotation $\beta_{i}$ with respect to the $X O Z$ plane.

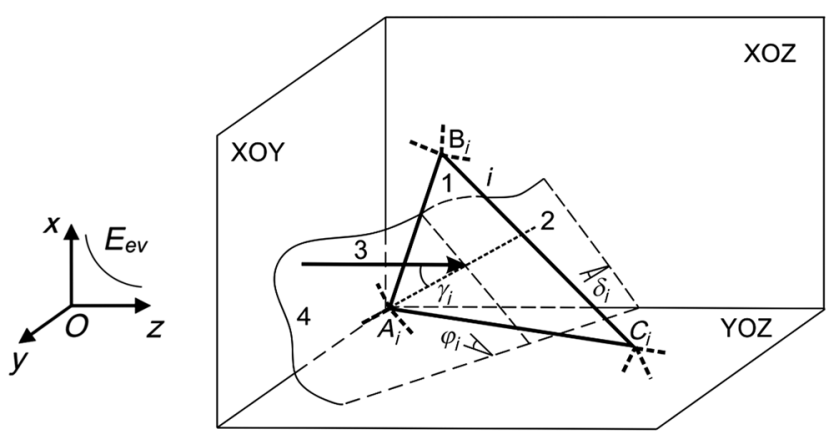

Fig. 3 Model decribing the incidence of an evanescent wave $E_{\mathrm{ev}}$ at the $i$ 'th facet $\left(A_{i} B_{i} C_{i}\right)$ of (1) the erythrocyte surface. $X Y Z$ are the coordinates determined by the condition of formation of an evanescent wave, corresponding to Fig. 1. (2) normal at the point of incidence of the beam 3 at facet 1 . The angle of incidence of the beam at the facet, $\gamma_{i}$, determines the plane of incidence 4 making angle $\varphi_{i}$ with the $Y O Z$-plane and angle $\delta_{i}$ with the $X O Y$-plane. 
Let us arbitrarily consider the $i$ 'th facet whose apexes are determined as $A_{i}\left(x_{A i}, y_{A i}, z_{A i}\right), B_{i}\left(x_{B i}, y_{B i}, z_{B i}\right)$, and $C_{i}$ $\left(x_{C i}, y_{C i}, z_{C i}\right)$. Then, the plane containing this facet can be determined as $a_{i} x+b_{i} y+c_{i} z+d_{i}=0$, where the coefficients $a_{i}$, $b_{i}, c_{i}, d_{i}$ are

$$
\begin{aligned}
a_{i}= & \left(y_{A_{i}}-y_{C_{i}}\right)\left(z_{B_{i}}-z_{A_{i}}\right)+\left(y_{B_{i}}-y_{A_{i}}\right)\left(z_{C_{i}}-z_{A_{i}}\right), \\
b_{i}= & \left(x_{C_{i}}-x_{A_{i}}\right)\left(z_{B_{i}}-z_{A_{i}}\right)+\left(x_{A_{i}}-x_{B_{i}}\right)\left(z_{C_{i}}-z_{A_{i}}\right), \\
c_{i}= & \left(x_{A_{i}}-x_{B_{i}}\right)\left(y_{A_{i}}-y_{C_{i}}\right)+\left(x_{C_{i}}-x_{A_{i}}\right)\left(y_{A_{i}}-y_{B_{i}}\right), \\
d_{i}= & \left(x_{C_{i}}-x_{A_{i}}\right)\left(y_{B_{i}} z_{A_{i}}-y_{A_{i}} z_{B_{i}}\right)+\left(y_{A_{i}}-y_{C_{i}}\right)\left(x_{B_{i}} z_{A_{i}}-x_{A_{i}} z_{B_{i}}\right) \\
& +\left(z_{C_{i}}-z_{A_{i}}\right)\left(x_{B_{i}} y_{A_{i}}-x_{A_{i}} y_{B_{i}}\right) .
\end{aligned}
$$

Here, the normal vector for the specified facet is determined by $\vec{n}_{i}\left(a_{i}, b_{i}, c_{i}\right)$. Then, the angle of inclination for the specified facet, $\alpha_{i}$, is given by $\cos \alpha_{i}=\frac{\left|a_{i}\right|}{\sqrt{a_{i}^{2}+b_{i}^{2}+c_{i}^{2}}}$, and the angle of rotation by $\cos \beta_{i}=\frac{\left|c_{i}\right|}{\sqrt{a_{i}^{2}+b_{i}^{2}+c_{i}^{2}}}$.

An evanescent wave propagating along the $O Z$ direction reaches the specified $i$ 'th facet and makes some angle $\gamma_{i}$ with the normal to this facet at the point of incidence of a beam (Fig. 3). If the vector determining the direction of propagation of the incident beam is determined as $\vec{b}\left(0,0, c_{i}\right)$, then $\cos \gamma_{i}=\frac{\left|c_{i}\right|}{\sqrt{a_{i}^{2}+b_{i}^{2}+c_{i}^{2}}}$.

The three coordinates for the specified facet determine the point of incidence of a beam onto the facet, being arbitrarily chosen. Consequently, the normal and the incident beam determine the plane of incidence, and one can use the Fresnel equations to compute the components of the electrical field of the reflected and refracted waves, and to determine the angle of incidence, $\gamma_{i}$, between the beam and the surface normal. Correspondingly, one can estimate the refraction angles, $\gamma_{i}^{t}$, for the incident beam at the each facet. The orientation of the plane of incidence with respect to the horizontal $Y O Z$ plane, i.e., the angle $\varphi_{i}$, can be estimated by $\cos \varphi_{i}=\frac{\left|b_{i} \cdot c_{i}\right|}{\sqrt{a_{i}^{2} \cdot c_{i}^{2}+b_{i}^{2} \cdot c_{i}^{2}}}$, calculated during the computer simulation. Correspondingly, one can determine the orientation of the plane of incidence with respect to the vertical $X O Z$ plane, i.e., the angle $\delta_{i}$, giving $\cos \delta_{i}=\frac{\left|a_{i} \cdot c_{i}\right|}{\sqrt{a_{i}^{2} \cdot c_{i}^{2}+b_{i}^{2} \cdot c_{i}^{2}}}$. Note, transition from one facet to another is accompanied by the change of energy of an evanescent wave proportional to $\cos \alpha_{i}$.

An evanescent wave, being excited above the interface, can be described by the following equation: ${ }^{24}$

$$
\vec{E}_{\mathrm{ev}}=E\left(\begin{array}{c}
\frac{1}{\sqrt{1+|m|^{2}}} \\
\frac{m}{\sqrt{1+|m|^{2}}} \frac{k}{k_{z}} \\
-i \frac{1}{\sqrt{1+|m|^{2}}} \frac{\kappa}{k_{z}}
\end{array}\right) \exp (-\kappa x) \exp \left[i\left(k_{z} z-\omega t\right)\right]
$$

Here $k_{z}=k \frac{n_{\mathrm{pr}}}{n_{\mathrm{sol}}} \sin \gamma$ is the propagation constant; $\kappa=k \sqrt{\left(\frac{n_{\mathrm{pr}}}{n_{\mathrm{sol}}}\right)^{2} \sin ^{2} \gamma-1}$ is the exponential decay rate; $m=\frac{T_{\perp}}{T_{/ /}} m_{1}$ is the state of polarization of an evanescent wave, ${ }^{25}$ where $m_{1}$ is the state of polarization of the probing beam impinging on the interface "plate-air," being equal to unity for linear polarization with the azimuth of polarization $45 \mathrm{deg} ; \gamma$ is the incidence angle on the surface, where total internal refection takes place; and $k$ is the wavenumber for the incident wave. The electrical field strength of an evanescent wave is $E=k_{z} / k \sqrt{\frac{\mu_{1}}{\mu}} T E_{0}$, where $T=\frac{\sqrt{\left|T_{/ /}\right|^{2}+\left|m_{1}\right|^{2}\left|T_{\perp}\right|^{2}}}{\sqrt{1+\left|m_{1}\right|^{2}}} \exp \left[i \arg T_{/ /}\right]$ is the transmission coefficient, ${ }^{26} T_{/ /}$and $T_{\perp}$ are the Fresnel transmission coefficients, and $E_{0}$ and $\omega$ are the strength and frequency of the electrical field of the incident wave, respectively.

At the coordinates $X Y Z$, for the plane of incidence one can write the components of the electrical vector of the reflected and refracted waves following the Fresnel equations. Therefore, for the reflected wave and the $i$ 'th facet one has

$$
\begin{aligned}
& {\left[E_{\mathrm{evx}}^{r}\right]_{i} }=\left[r_{/ /}\right]_{i} \cdot\left[E_{\mathrm{ev} / /]_{i}} \sin \gamma_{i} \sin \varphi_{i},\right. \\
& {\left[E_{\mathrm{evy}}^{r}\right]_{i} }=\left[r_{\perp}\right]_{i}\left[E_{\mathrm{ev} \perp}\right]_{i} \cos \varphi_{i} \sin \delta_{i}, \\
& {\left[E_{\mathrm{evz}}^{r}\right]_{i} }=-\left[r_{/ /}\right]_{i} \cdot\left[E_{\mathrm{ev} / /]_{i}} \cos \gamma_{i} \cos \varphi_{i} \cos \delta_{i} .\right. \\
& \text { Here }\left\{\begin{array}{l}
{\left[r_{/ /}\right]_{i}=\frac{n_{\mathrm{er}} \cos \gamma_{i}-n_{\mathrm{sol}} \cos \gamma_{i}^{t}}{n_{e r} \cos \gamma_{i}+n_{\mathrm{sol}} \cos \gamma_{i}^{t}}} \\
{\left[r_{\perp}\right]_{i}=\frac{n_{\mathrm{sol}} \cos \gamma_{i}-n_{\mathrm{er}} \cos \gamma_{i}^{t}}{n_{\mathrm{sol}} \cos \gamma_{i}+n_{\mathrm{er}} \cos \gamma_{i}^{t}}}
\end{array}\right. \text { are the reflection coeffi- }
\end{aligned}
$$
cients providing computation of energy of the reflected evanescent wave at the $i$ 'th facet, i.e., the share of energy that is not involved in the formation of the components of the optical force, and determination of the part of the energy of the refracted wave resulting in spatial motion of the erythrocyte.

For the refracted evanescent wave, the components of the complex amplitude are written as

$$
\begin{aligned}
& {\left[E_{\mathrm{evx}}^{t}\right]_{i}=\left[t_{/ / /}\right]_{i} \cdot\left[E_{\mathrm{ev} / /]_{i}} \cdot \sin \gamma_{i}^{t} \sin \varphi_{i},\right.} \\
& {\left[E_{\mathrm{evy}}^{t}\right]_{i}=\left[t_{\perp}\right]_{i} \cdot\left[E_{\mathrm{ev} \perp}\right]_{i} \cos \varphi_{i} \sin \delta_{i} .} \\
& {\left[E_{\mathrm{evz}}^{t}\right]_{i}=-\left[t_{/ / /}\right]_{i} \cdot\left[E_{\mathrm{ev} / /]_{i}} \cdot \cos \gamma_{i}^{t} \cos \varphi_{i} \cos \delta_{i} .\right.} \\
& \text { Here, }\left\{\begin{array}{l}
{\left[t_{/ /}\right]_{i}=\frac{2 n_{\mathrm{sol}} \cos \gamma_{i}}{n_{\mathrm{er}} \cos \gamma_{i}+n_{\mathrm{sol}} \cos \gamma_{i}^{t}} \text { are the refraction coeffi- }} \\
{\left[t_{\perp}\right]_{i}=\frac{2 n_{\mathrm{sol}} \cos \gamma_{i}}{n_{\mathrm{er}} \cos \gamma_{i}^{t}+n_{\mathrm{sol}} \cos \gamma_{i}}}
\end{array}\right.
\end{aligned}
$$
cients for the parallel and perpendicular components of an evanescent wave that impinges onto the $i$ 'th facet of an erythrocyte. $\left[E_{\mathrm{ev} / /}\right]_{i}\left(\left[E_{\mathrm{ev} \perp}\right]_{i}\right)$ are the components of the electrical vector of the incident evanescent wave at the plane of incidence, at the $i$ 'th facet. Hence,

$$
\begin{aligned}
{\left[E_{\mathrm{ev} / /}\right]_{i} } & =E_{\mathrm{ev}} \cdot\left(\cos \varphi_{i} \sin ^{2} \delta_{i} \sin \gamma_{i}+\cos \varphi_{i} \cos ^{2} \delta_{i} \sin \gamma_{i}\right. \\
& \left.-\sin ^{2} \varphi_{i} \cos \gamma_{i}\right) \\
{\left[E_{\mathrm{ev} \perp}\right]_{i} } & =E_{\mathrm{ev}} \cdot\left(\cos \varphi_{i} \sin ^{2} \delta_{i} \cos \alpha_{i}+\cos \varphi_{i} \cos ^{2} \delta_{i} \sin \alpha_{i}\right. \\
& \left.+\sin ^{2} \varphi_{i} \sin \alpha_{i}\right)
\end{aligned}
$$

are the fields for the $i$ 'th facet.

Determination of the optical force in the $Y O Z$ plane with the components in the longitudinal and transversal directions (what is typical for an evanescent wave) for the azimuth of polarization of the incident wave $\pm 45 \mathrm{deg}$ requires computation of the density of the spin and orbital angular momenta at the directions $O Y$ and $O Z$. The components are initially computed for each facet and then the corresponding components are summed. The magnitudes of the components of the refracted evanescent wave at each facet are used for obtaining the estimation of the spin orbital momentum density and the orbital angular momentum density in the longitudinal and transversal directions. The spin angular momentum density in this case is expressed as in 
Refs. 25 and 26, $\left[p_{s}\right]_{i}=\frac{1}{16 \pi \omega} \nabla \times \operatorname{Im}\left[\left[\vec{E}_{\mathrm{ev}}^{t *}\right]_{i} \times\left[\vec{E}_{\mathrm{ev}}^{t}\right]_{i}\right]$. Here, $\left[\vec{E}_{\mathrm{ev}}^{t}\right]_{i}$ is the transmitted evanescent wave interacting with the $i$ 'th facet of the erythrocyte. This expression contains both the longitudinal and transversal components. ${ }^{22}$ The orbital angular momentum is given by $\left[p_{o}\right]_{i}=\frac{1}{8 \pi \omega} \operatorname{Im}\left[\left[\vec{E}_{\mathrm{ev}}^{t *}\right]_{i} \cdot(\nabla)\left[\vec{E}_{\mathrm{ev}}^{t}\right]_{i}\right]$. The orbital (canonical) angular momentum caused by light pressure determines a force transferred to a cell in the direction of propagation of an evanescent wave.

Thus, the resulting momentum density in the $O Z$-direction is given as

$$
\begin{aligned}
{\left[p_{z}\right]_{i}=} & {\left[p_{o z}\right]_{i}+\left[p_{s z}\right]_{i}=\frac{[t / /]_{i}^{2} \cdot \cos ^{2} \gamma_{i}^{t} \cdot \cos \delta_{i}^{2} \cdot A^{2}}{8 \pi \omega} } \\
& \times\left[\left(k_{z}+\frac{m^{2} k^{2}}{k_{z}}+\frac{\kappa^{2}}{k_{z}}\right)-2 \frac{\kappa^{2}}{k_{z}}\right] \exp (-2 \kappa x),
\end{aligned}
$$

and the transversal momentum caused by the vertical spin becomes $\left[p_{y}\right]_{i}=\left[p_{s y}\right]_{i}=\frac{\left[t_{\perp}\right]_{i}^{2} \cdot \sin \delta_{i}^{2} \cdot A^{2}}{4 \pi \omega} \frac{k \kappa}{k_{z}} \operatorname{Im} m \exp (-2 \kappa x)$, where $A=E \frac{\cos \varphi_{i}}{\sqrt{1+|m|^{2}}}$.

Let us compute the optical force $\vec{F}_{i}$ acting on each facet of an erythrocyte. We use the Mie light-scattering theory, under which approximation, ${ }^{26-28} \vec{F}_{i}=\Delta \vec{p}_{i} S_{i}$, where $\Delta p_{i}$ is the change of momentum density at the corresponding facet $S_{i}$ interacting with the evanescent wave for $i=1$ to $N$, where $N$ is the number of facets. Thus, obtained $\Delta\left[p_{y}\right]_{i}$ and $\Delta\left[p_{z}\right]_{i}$ make it possible to calculate components of the optical force $\left[F_{Y}\right]_{i}$ and $\left[F_{Z}\right]_{i}$.

The refracted evanescent wave in the $O X$-direction for each facet $i$ also forms some component of the optical force in this direction, which can be computed using the relation: $\left[F_{x}\right]_{i}=\int_{\sigma}\left\langle\left[\stackrel{\leftrightarrow}{T}_{x}\right]_{i}\right\rangle \hat{n} \mathrm{~d} S_{i}$, where $\left[\stackrel{\leftrightarrow}{T}_{x}\right]_{i}$ is the component of the time-averaged Maxwell's stress-vector

$$
\begin{aligned}
\left\langle\stackrel{T}{T}_{i}\right\rangle= & \frac{\varepsilon_{0}}{2}\left\{\left[\vec{E}_{\mathrm{ev}}^{t}\right]_{i}\left[\vec{E}_{\mathrm{ev}}^{t}\right]_{i}^{*}+c^{2}\left[\vec{B}_{\mathrm{ev}}^{t}\right]_{i}\left[\vec{B}_{\mathrm{ev}}^{t}\right]_{i}^{*}\right. \\
& \left.-\frac{1}{2}\left[\left|\left[\vec{E}_{\mathrm{ev}}^{t}\right]_{i}\right|^{2}+c^{2}\left|\left[\vec{B}_{\mathrm{ev}}^{t}\right]_{i}\right|^{2}\right] I\right\}
\end{aligned}
$$

computed for the $i$ 'th facet in the $O X$-direction. Here, $I$ is the unit matrix, $\hat{n}$ is the surface-normal vector, $B_{\mathrm{ev}}^{t}=\sqrt{\varepsilon \varepsilon_{0}} E_{\mathrm{ev}}^{t}$ is the magnetic inductance vector for the evanescent wave, and $\varepsilon$ is the media permittivity.

Inhomogeneous in intensity refracted evanescent wave caused by the action of the gradient force $\left[\vec{F}_{\mathrm{grad}}\right]_{i}=-\frac{1}{2} n \nabla\left[E_{\mathrm{ev}}^{t}\right]_{i}^{2}$ is connected with the direction of propagation of the beam refracted at the $i$ 'th facet of the beam. The total component of the optical force in the $O X$-direction for the $i$ 'th facet is $\left[F_{X}\right]_{i}=\left[F_{x}\right]_{i}+\left[F_{\operatorname{grad} x}\right]_{i}$. The next calculation step consists in recalculation of the found set of optical force components for each of the specified facets, $\left[F_{X}\right]_{i},\left[F_{Y}\right]_{i},\left[F_{Z}\right]_{i}$, into the resulting components, by summation of the corresponding force components over all facets of the erythrocyte surface, $F_{m}=\sum_{i}^{N}\left[F_{m}\right]_{i}, m=X, Y, Z$.

The computed results show that the magnitude of the component of the force $F_{X}$ is not large enough to provide the vertical motion of an erythrocyte, which is by several orders of magnitude smaller than the magnitude of the longitudinal component, and therefore has no influence on the spatial motion of a cell.

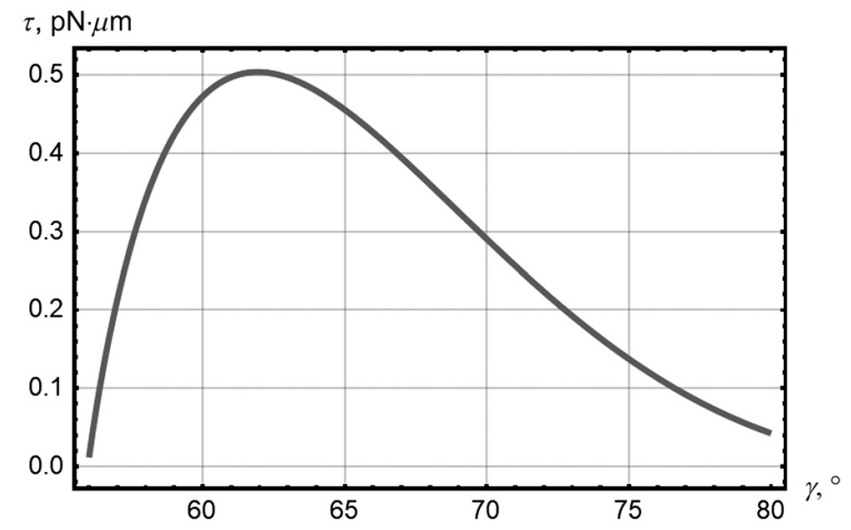

Fig. 4 Dependence of the magnitude of the torque resulting from action of an evanescent wave on an erythrocyte as a function of the angle of incidence of the laser beam onto the prism.

The estimated ratio of the components of the optical force in the longitudinal and transversal directions also shows that the longitudinal force component (in the $O Z$-direction) is much larger than the transversal component. Thus, a rectilinear motion of investigated erythrocyte $i$ observed in the longitudinal $O Z$ direction. For visualization of such a motion of a cell in the $O Y$-direction, illumination of an erythrocyte by one wave is also not a sufficient condition, forcing the search for alternative approaches for demonstration of such motions. The action of an evanescent wave on erythrocyte in this model experiment also manifests itself in an observed rotational motion of a cell with respect to its axis of symmetry.

Let us use the relation $\tau_{i}=\int\left(\vec{r}_{i} \times\left\langle\overleftrightarrow{T}_{i}\right\rangle\right) \cdot \hat{n} \mathrm{~d}^{2} r_{i}$ for computing the torque of an erythrocyte (Fig. 1) for the $i^{\prime}$ th facet. Here, $\stackrel{\leftrightarrow}{T}_{i}$ is the above represented Maxwell's stress-tensor [Eq. (3)] computed for the specified facet, $\vec{r}_{i}$ is the position vector drawn from the axis of rotation of an erythrocyte, $O O^{\prime}$, to the point of application of the optical force of an evanescent wave at the specified facet of the cell surface. The resulting torque of the force, $\tau$, is equal to the sum of momenta of the force transferred to separate parts (facets) of an erythrocyte accounting for the direction of rotation, i.e., $\tau=\sum_{i}^{N} \tau_{i}$. The results of calculation are presented in Fig. 4.

\section{Modeling of Controlled Transversal Motion of Erythrocyte}

For observation of the controlled transversal motion of an erythrocyte in the YOZ-plane (which is possible only if the transversal components of the evanescent wave are active), we propose to implement the model arrangement shown in Fig. 5, where two oppositely directed evanescent waves of the same amplitude and frequency are formed above the prism. In the model experiment, the longitudinal components of the optical force of the evanescent waves onto the erythrocyte are compensated, so that only the transversal components are added. Let us emphasize, the discussed effect only becomes possible if one uses two linearly polarized incident waves with the azimuths of polarization \pm 45 deg with respect to the plane of incidence, so that two oppositely directed evanescent waves are of the same elliptical polarization.

In accordance with the above represented scheme for computation of the optical force generated by the evanescent wave, 


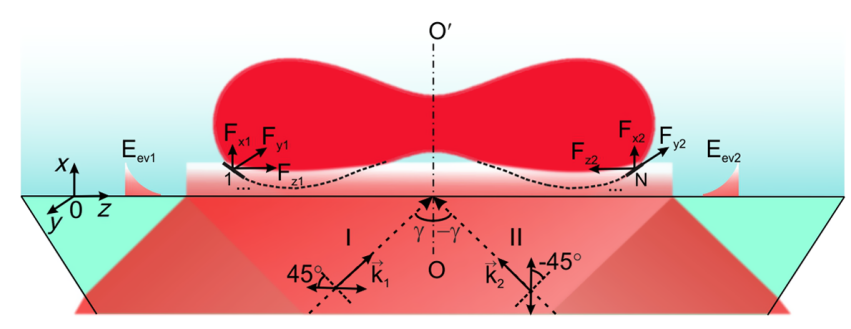

Fig. 5 Two linearly polarized beams I and II with azimuths of polarization \pm 45 deg with respect to the plane of incidence are incident on the prism at the angle $\gamma$. Here, $\vec{k}_{1}, \vec{k}_{2}$ are the wavevectors of the incident beams; $F_{x 1}, F_{x 2}, F_{y 1}, F_{y 2}, F_{z 1}$, and $F_{z 2}$ are the components of the optical force of the evanescent waves $E_{\mathrm{ev} 1}$ and $E_{\mathrm{ev} 2} ; O O^{\prime}$ is the axis of symmetry of an erythrocyte. $N=1 . . i$ is the number of simulating facets.

one determines the force components $F_{m 1}$ and $F_{m 2}$ for the first (I) and second (II) (Fig. 5) evanescent waves acting on the erythrocyte. As the preliminary simulation shows, the incidence of the beams onto the surface of a prism, where total internal reflection appears, must be symmetrical with respect to the axis of symmetry of an erythrocyte $O O^{\prime}$.

Let the polarizations of the two beams be the same, i.e., the azimuths of polarization of both beams are equal to $+45 \mathrm{deg}$ or $-45 \mathrm{deg}$. Thus, for the symmetrical incidence of the beams, both the total longitudinal component of the optical force, $F_{Z}=F_{Z 1}+F_{Z 2}$, and the total transversal component, $F_{Y}=F_{Y 1}+F_{Y 2}$, become equal zero. $F_{Y 1, Y 2}, F_{Z 1, Z 2}$ are the transversal and longitudinal components, respectively, of the evanescent waves excited by the linearly polarized incident waves with azimuths of polarization $\pm 45 \mathrm{deg}$. Evanescent waves formed above the prism surface are elliptically polarized, with the same direction of rotation of the electric-field vector, each of which has torque, as has been shown above (cf. Figs. 1 and 4), which is inherent in the vertical spin of an evanescent wave. Such action results in the total torque that is twice as large for the chosen angles, see Fig. 4.

Formation of the transversal component of the optical force and demonstration of its directional action presumes the use of two linearly polarized incident beams whose azimuths of polarization are orthogonal, viz. $+45 \mathrm{deg}$ and $-45 \mathrm{deg}$ (cf. Fig. 5). Elliptically polarized evanescent waves with the opposite directions of rotation of the electric field vectors compensate the resulting rotational motion of a cell and provide the condition for observation of a rectilinear motion of an erythrocyte.

In accordance with the conditions of modeling, for compensated longitudinal component $F_{Z}=F_{Z 1}+F_{Z 2}$, one observes strengthening of the transversal component, which can be visualized experimentally. The magnitude of the transversal component and its influence on the characteristics of motion of a cell depend on the angle of incidence of waves on the erythrocyte surface and determine the velocity of a cell's spatial motion. In Fig. 6, we represent the graphic demonstration of changing transversal component of the force depending on the angle of incidence. Here, $F_{Y}=F_{Y 1}+F_{Y 2}$. This enables variation of the spatial position of an erythrocyte. Let us assume that the transversal forces formed because of the action of an evanescent wave do not cause deformation of a moving erythrocyte. The maximal magnitude of the resulting optical force occurs for an angle of incidence of $63 \mathrm{deg}$. The parameters of erythrocyte shape used in simulation have been obtained from the experimental data. ${ }^{20}$

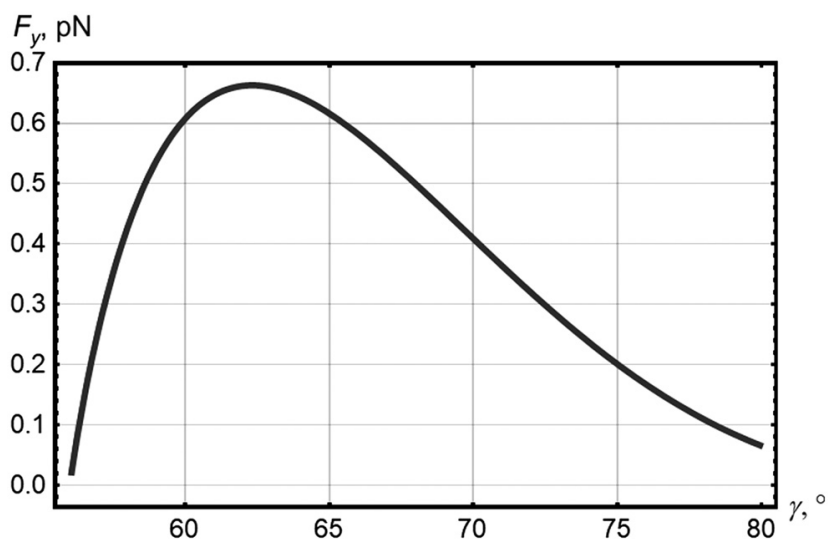

Fig. 6 Evolution of the transversal component of the optical force at an evanescent feild.

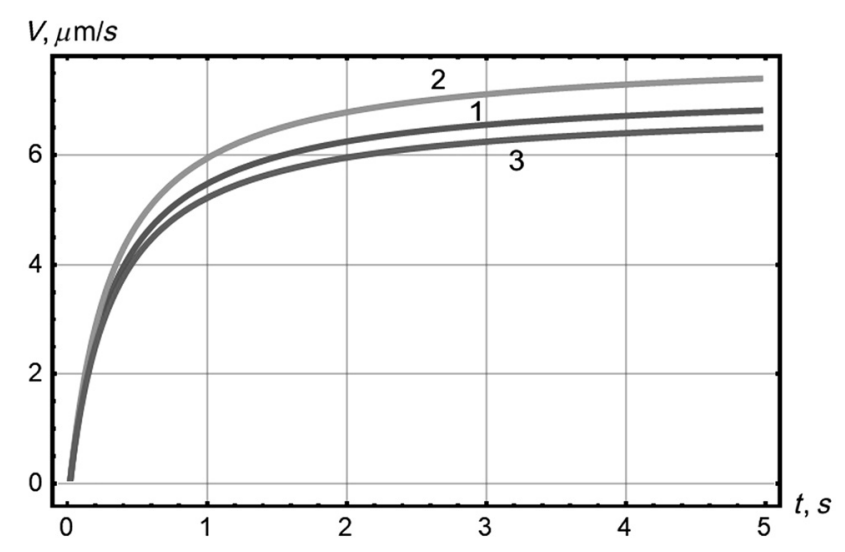

Fig. 7 Velocity of an erythrocyte in the transversal direction depending on the angle of illumination: $1-60 \mathrm{deg} ; 2-62 \mathrm{deg} ; 3-66 \mathrm{deg}$.

In the previous, we have argued for the feasibility for control of spatial (transversal) motion of the studied microobject. Transversal motion of an erythrocyte in blood plasma is caused by the action of the resulting force $\vec{F}=\vec{F}_{\text {opt }}+\vec{F}_{\text {st }}$, where $F_{\text {opt }}=F_{Y}$ is the optical force. At low Reynolds numbers, the damping factor or drag coefficient can be estimated by approximating the disk shape of erythrocyte by a sphere of the same cross-sectional area, ${ }^{29}$ then $F_{\text {st }}=3 \pi \eta D v$ is the Stokes force. Here, $\eta=1.236 \times 10^{-3} \mathrm{~Pa} \cdot \mathrm{s}$ is the dynamic viscosity of blood plasma, $D$ is the diameter of the corresponding cross sectional area of the cell, and $v$ is the velocity of an erytrhocyte. While $m \frac{d v}{d t}=F+3 \pi \eta D v$, one can determine velocity of erythrocyte in a solution at the transversal direction as a function of time (Fig. 7), by comparing the theoretical and experimental results.

In the course of time, the velocity of an erythrocyte is experimentally detected while changing the angle of illumination.

\section{Experimental Demonstration of Transversal Motion of Erythrocytes into Evanesent Field}

The results of computer simulation of moving erythrocytes in an evanescent field will now be supported experimentally. A blood plasma $^{30}$ is prepared by centrifugation of fresh venous blood at room temperature. Initially, a whole blood $(1500 \mathrm{~g})$ is centrifuged during $10 \mathrm{~min}$, which results in separation of sedimented 


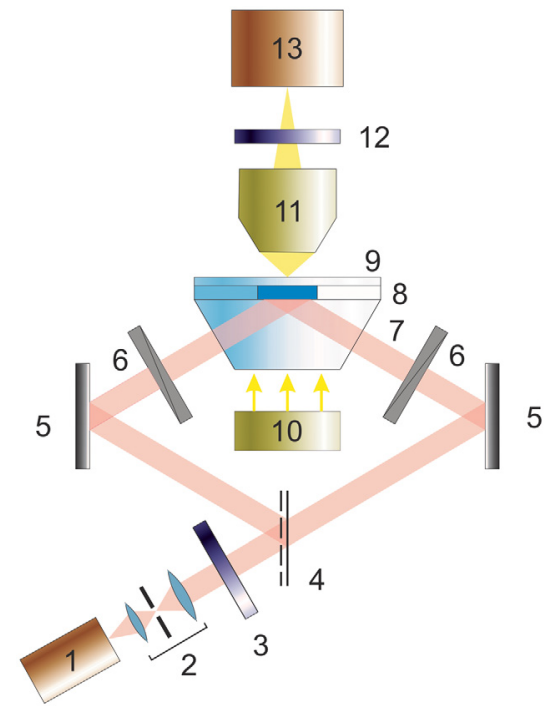

Fig. 8 Optical arrangement for study of moving erythrocytes in an evanescent field: (1) laser; (2) beam expander with a spatial filter; (3) quarter-wave plate; (4) beam splitter; (5) mirrors; (6) polarizers; (7) total reflection prism; (8) cuvette with erythrocytes; (9) LED display; (10) microobjective; (11) light filter; and (12) CCD camera.

erythrocytic masses from plasma. Further, a second centrifugation $(1500 \mathrm{~g})$ during $10 \mathrm{~min}$ is carried out at room temperature for removing residual cells, such as thrombocytes. Then, blood from a finger $(0.5 \mu \mathrm{g})$ is diluted in $1 \mathrm{ml}$ of blood plasma. The prepared suspension $(50 \mu \mathrm{l})$ is placed in a hermetic cuvette consisting of a cover glass of $0.15 \mathrm{~mm}$ thickness and the total reflection prism at the distance $0.05 \mathrm{~mm}$. To avoid adhesion of erythrocytes, a cover glass and the prism surface are sequentially washed out in a solution of surface-active material, in $96 \%$ ethyl alcohol, then in deionized water, and finally in $3 \%$ solution of hydrogen peroxide, and then dried and washed out in $2 \%$ solution of human albumin.

The optical arrangement for the experimental demonstration of erythrocyte motion in an evanescent field is shown in Fig. 8.

The radiation from a semiconductor laser 1 with wavelength $\lambda=980 \mathrm{~nm}$ and power $1 \mathrm{~W}$ passes the beam expander 2 consisting of two objectives and a micron-scale pinhole as the spatial frequency filter. We varied the radiation power from 5 to $500 \mathrm{~mW}$. The beam with a plane wavefront passes a quarter-wave plate 3 and becomes circularly polarized. After beam splitter 4, two beams of equal intensities are reflected by the mirrors 5 to the total reflection prism 7. Polarizers 6 form the orthogonally linearly polarized beams with the azimuths of polarization $45 \mathrm{deg}$, which fall on the interface glass-blood plasma. In a cuvette 8 with erythrocytes, two oppositely directed evanescent waves of equal intensities are formed. Moving erythrocytes are observed in white light from a LED display 9 using a microobjective 10 (magnification $60^{\mathrm{X}}$ ) and CCD-camera 12. Light filter 11 cuts off radiation from the IR laser.

We studied rectilinear motion of an erythrocyte in one evanescent wave, when the right beam in Fig. 8 is blocked (Fig. 9), and into two oppositely directed beams (Fig. 10). The motion of an erythrocyte in one evanescent beam is shown in Fig. 9 for the frame frequency $2 \mathrm{~Hz}$ and radiation power $100 \mathrm{~mW}$. The speed of motion of an erythrocyte is about $35 \mu \mathrm{m} / \mathrm{s}$. An erythrocyte travels along the direction of propagation of the evanescent field. At the same time, one observes also some transversal motion of an erythrocyte with the speed $4 \mu \mathrm{m} / \mathrm{s}$, approximately, which is caused by the transversal component of the force $F_{y}$ influencing erythrocyte in the evanescent field. This situation was previously theoretically predicted.

Motion of an erythrocyte in opposite evanescent waves is shown in Fig. 10.

The frame frequency equals $1 \mathrm{~Hz}$. One observes only transversal motion of an erythrocyte with a speed $6 \mu \mathrm{m} / \mathrm{s}$, approximately. The longitudinal moving is compensated by the opposite beams, while the transversal moving for linearly polarized beams with the azimuths of polarization $\pm 45 \mathrm{deg}$ doubles, in agreement with the theoretical prediction. One observes correlation of the results of computer simulation and the experimental study with a discrepancy not exceeding $30 \%$.

Such a discrepancy is explained by the influence of various kinds of factors on the purity of the experiment. More specifically, the experimental solution with RBC was insufficiently clean, i.e., the presence of inhomogeneities giving rise to scattering was observed. This determined the second action from the evanescent wave on the object. In addition, our experimental realization assumed the change of the azimuth of polarization of the evanescent wave from $\pm 45 \mathrm{deg}$ (that was about $2 \%$ to $3 \%$ ), which determined one of the possible causes of the experimental error as well.

\section{Conclusion}

A specially chosen theoretical model for exciting an evanescent wave and calculation of its impact on an erythrocyte has provided the conditions for rectilinear and rotational motion of
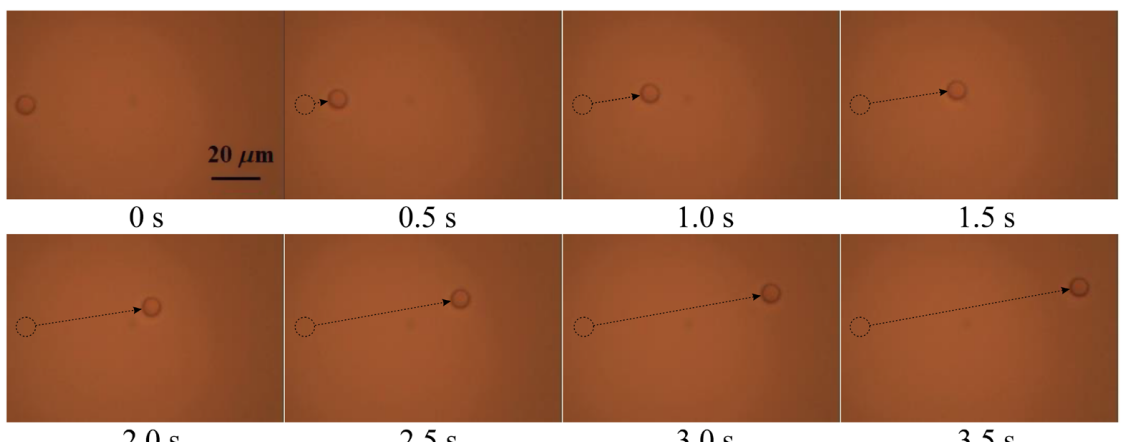

$\begin{array}{llll}2.0 \mathrm{~s} & 2.5 \mathrm{~s} & 3.0 \mathrm{~s} & 3.5 \mathrm{~s}\end{array}$

Fig. 9 Motion of an erythrocyte in one evanescent wave. The frame frequency is $2 \mathrm{~Hz}$. The dotted circle indicates the initial position of the erythrocyte, and the dotted arrow indicates the direction of the erythrocyte motion. 

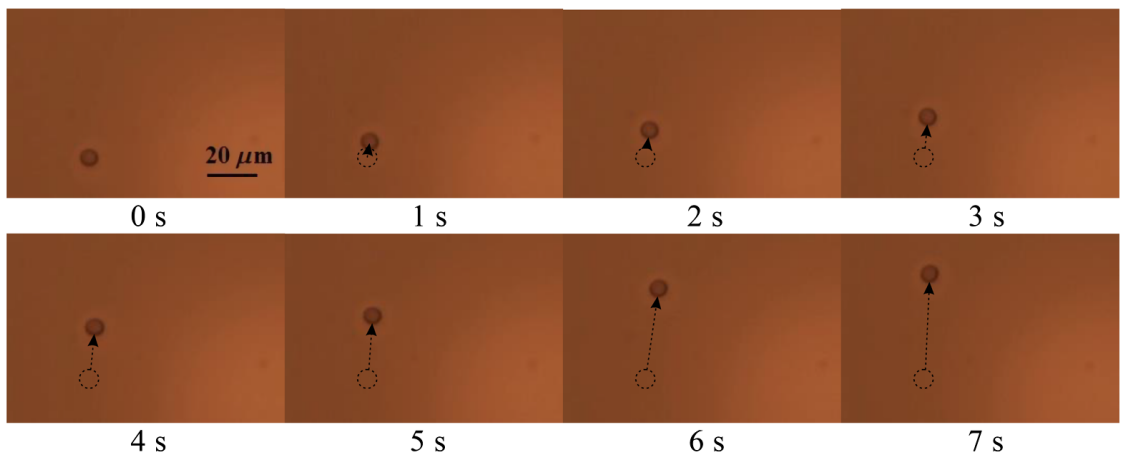

Fig. 10 Motion of an erythrocyte in opposite evanescent waves. The frame frequency is $1 \mathrm{~Hz}$. The dotted circle indicates the initial position of the erythrocyte, and the dotted arrow indicates the direction of the erythrocyte motion.

an isolated erythrocyte. It has been shown that, for the specified experimental conditions, the longitudinal motion of an erythrocyte is compensated, accompanied by an increasing transversal component of the optical force manifesting itself in controlled transversal red blood cell motion. The speed of transversal motion of an erythrocyte has been evaluated both theoretically and experimentally. Specifically, the discrepancy between the theoretical and experimental results does not exceed $30 \%$.

\section{Disclosures}

The authors have no relevant financial interests in this article and no potential conflicts of interest to disclose.

\section{References}

1. H. Xin and B. Li, "Targeted delivery and controllable release of nanoparticles using a defect-decorated optical nanofiber," Opt. Express 19(14), 13285-13290 (2011).

2. K. Dholakia and P. Reece, "Optical micromanipulation takes hold," Nano Today 1(1), 18-27 (2006)

3. M. D. Wang et al., "Stretching DNA with optical tweezers," Biophys. J. 72(3), 1335-1346 (1997).

4. C. Bustamante, Z. Bryant, and S. B. Smith, "Ten years of tension: single-molecule DNA mechanics," Nature 421(6921), 423-427 (2003).

5. D. Altman, H. L. Sweeney, and J. A. Spudich, "The mechanism of myosin VI translocation and its load-induced anchoring," Cell 116(5), 737-749 (2004).

6. C. L. Asbury, A. N. Fehr, and S. M. Block, "Kinesin moves by an asymmetric hand-over-hand mechanism," Science 302(5653), 2130-2134 (2003).

7. A. Ashkin, "Acceleration and trapping of particles by radiation pressure," Phys. Rev. Lett. 24, 156-159 (1970).

8. M.-Ch. Zhong et al., "Trapping red blood cells in living animals using optical tweezers," Nat. Commun. 4, 1768 (2013).

9. B. Akiyoshi et al., "Tension directly stabilizes reconstituted kinetochore-microtubule attachments," Nature 468, 576-579 (2010).

10. X. B. Wei, B. J. Tromberg, and M. D. Cahalan, "Mapping the sensitivity of T cells with an optical trap: Polarity and minimal number of receptors for $\mathrm{Ca}^{2+}$ signalling," Proc. Natl Acad. Sci. U. S. A. 96, 8471-8476 (1999)

11. Y.-Z. Yoon and P. Cicuta, "Optical trapping of colloidal particles and cells by focused evanescent fields using conical lenses," Opt. Express 18(7), 7076-7084 (2010).

12. G. D. Byrne et al., "Total internal reflection microscopy for live imaging of cellular uptake of sub-micron non-fluorescent particles," J. Microsc. 231(1), 168-179 (2008)

13. G. Brambilla et al., "Optical manipulation of microspheres along a subwavelength optical wire," Opt. Lett. 32(20), 3041-3043 (2007).

14. H. B. Xin et al., "Photothermal trapping of dielectric particles by optical fiber-ring," Opt. Express 19(3), 2711-2719 (2011).
15. H. X. Lei et al., "Photophoretic assembly and migration of dielectric particles and Escherichia coli in liquids using a subwavelength diameter optical fiber," Lab Chip 11(13), 2241-2246 (2011).

16. O. V. Angelsky et al., "Influence of evanescent wave on birefringent microplates," Opt. Express 25(3), 2299-2311 (2017).

17. L. Liu et al., "Elliptical orbits of microspheres in an evanescent field," Proc. Natl. Acad. Sci. U. S. A. 114(42), 11087-11091 (2017).

18. V. Tuchin, Optical Biomedical Diagnostics, Vol. 1 (2017) (in Russian).

19. M. M. Kugeyko and D. A. Smunyov, "Determination of microphysical parameters of native erythrocytes on the results of measuring the optical characteristics of scattered radiation," Bull. Belorussian State Univ. 1(2), 73 (2016) (in Russian).

20. M. Gu, S. Kuriakose, and X. Gan, "A single beam near-field laser trap for optical stretching, folding and rotation of erythrocytes," Opt. Exp. 15(3), 1369-1375 (2007).

21. X. Chen et al., "Observation of spin and orbital rotation of red blood cell in dual-beam fibre-optic trap with transverse offset," J. Opt. 19, 055612 (2017).

22. M. A. Yurkin, "Modelling of light scattering by blood cells using the discrete dipoles method," PhD Thesis, Novosibirsk (2008) (in Russian).

23. S. C. Grover, R. C. Gauthier, and A. G. Skirtach, "Analysis of the behaviour of erythrocytes in an optical trapping system," Opt. Express 7(13), 533-539 (2000).

24. A. Hayata, J. P. Balthasar Muellera, and F. Capassoa, "Lateral chiralitysorting optical forces," Proc. Natl. Acad. Sci. U. S. A. 112(43), 1319013194 (2015).

25. K. Y. Bliokh, A. Y. Bekshaev, and F. Nori, "Dual electromagnetism: helicity, spin, momentum, and angular momentum," New J. Phys. 15, 033026 (2013).

26. M. Antognozzi et al., "Direct measurements of the extraordinary optical momentum and transverse spin-dependent force using a nanocantilever," Nat. Phys. 12, 731-735 (2016).

27. C. Yu. Zenkova, D. I. Ivanskyi, and T. V. Kiyashchuk, "Optical torques and forces in birefringent microplate," Opt. Appl. 47(3), 483-493 (2017).

28. C. Yu. Zenkova and D. I. Ivanskyi, "Non-trivial structure of optical momentum and optical forces inherent in evanescent waves," Proc. SPIE 10612, 1061207 (2018).

29. S. C. Grover, R. C. Gauthier, and A. G. Skirtach, "Analysis of the behaviour of erythrocytes in an optical trapping system," Opt. Express 7(13), 533-539 (2000)

30. A. Ahmad et al., "Quantitative phase microscopy of red blood cells during planar trapping and propulsion," Lab Chip 18, 3025-3036 (2018).

Oleg V. Angelsky is a director of the Institute of Physical, Technical, and Computer Sciences, Chernivtsi National University, Ukraine. He is a fellow of the Institute of Physics (United Kingdom), SPIE, OSA, and EOS, and a member of an advisory committee for EOS. He is the author of about 300 publications, including some chapters of international books, for which he was an editor. His research interests are in rough surface characterization, fractal optics, holography, polarization optics, correlation optics, and singular optics. 
Peter P. Maksimyak is a head of the Correlation Optics Department of Chernivtsi National University, Ukraine. $\mathrm{He}$ is the author/coauthor of about 250 publications, including some chapters of international books. His research interests are optical correlation and holographic diagnostics of random fields and objects, fractal optics, and singular optics.

Claudia Y. Zenkova is a vice director in international relations for the Institute of Physical, Technical and Computer Sciences, Chernivtsi National University, Ukraine. She is the author/coauthor of about 90 publications, including chapters for international books, mainly in polarization and singular optics, correlation optics of partially coherent and partially polarized light fields.

Andrew P. Maksimyak received his MSc degree in laser and optoelectronic technology from Chernivtsi University in 2009, and his PhD in optics and laser physics from Chernivtsi University in 2012. Since 2014, he has been a senior research fellow at the Chernivtsi National
University in the Correlation Optics Department. He is the coauthor of about 50 publications mainly in optical correlation diagnostics of random fields and objects, and singular optics.

Steen G. Hanson is a professor emeritus. at the Technical University of Denmark (DTU), Photonics Department. He received his PhD at DTU in 1974 and is former chairman of the Danish Optical Society and head of the laser section at Risø National Laboratory in Denmark. He was recognized by the United States Department of Commerce in the form of a certificate of recognition for outstanding performance in 1993, and appointed senior member of OSA, November 12, 2010. In September 2017, he was appointed honorary doctor at Chernivtsi University, Ukraine.

Dimitrov D. Ivanskyi received his MS degree in laser and optoelectronics techniques in 2015, his $\mathrm{PhD}$ in optics and laser physics in 2019 from Chernivtsi National University, Department of Optics, Publishing, and Printing, Chernivtsi, Ukraine. 